\title{
Experimental and theoretical radiative properties of odd-parity highly excited levels in Mo II
}

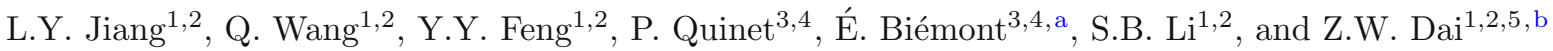 \\ 1 College of Physics, Jilin University, Changchun 130012, P.R. China \\ 2 Key Lab of Coherent Light, Atomic and Molecular Spectroscopy, Ministry of Education, Changchun 130012, P.R. China \\ 3 Astrophysics and Spectroscopy, University of Mons, 700 Mons, Belgium \\ 4 IPNAS, University of Liège, Sart Tilman, Bât. B15, 4000 Liège, Belgium \\ ${ }^{5}$ Institute of Atomic and Molecular Physics, Jilin University, Changchun 130012, P.R. China
}

Received 7 April 2012 / Received in final form 7 May 2012

Published online 10 July 2012 - (c) EDP Sciences, Società Italiana di Fisica, Springer-Verlag 2012

\begin{abstract}
Radiative lifetimes of 13 odd-parity levels of Mo II have been measured using time-resolved laser-induced fluorescence (TR-LIF) spectroscopy in a plasma produced by laser ablation. The investigated levels, within the energy range 48 022-63 $497 \mathrm{~cm}^{-1}$, were excited from the ground or appropriate metastable states with one-photon selective excitation. The uncertainties of the measured lifetime results are less than 10\%. Calculated lifetimes of Mo II, obtained using the relativistic Hartree-Fock (HFR) method including core-polarization effects, are found to agree well with the measured ones. New transition probabilities are deduced for 246 transitions in the wavelength range extending from 207.431 up to $510.813 \mathrm{~nm}$.
\end{abstract}

\section{Introduction}

Accurate radiative lifetimes can be used for obtaining oscillator strengths when they are combined with experimental or theoretical branching ratios [1]. Oscillator strengths are of great importance for the determination of elemental abundances in stellar photospheres [2]. In astrophysics, transitions of Mo II have been observed in stellar and interstellar spectra. For example, Jascheck and Brandi [3] and Cowley et al. [4] observed Mo II lines in two Ap stars of the Cr-Eu-Sr subgroup, while Adelman [5] confirmed the presence of Mo in at least 11 cool Ap stars of the same subgroup. In 1999, Brandt et al. [6] identified two Mo II resonance lines at 201.5 and $202 \mathrm{~nm}$ in the HgMn-type star $\chi$ Lupi using the Goddard high-resolution spectrograph on board the Hubble space telescope.

Presently, experimental transition probabilities and oscillator strengths for Mo II transitions emitted from highly excited levels are scarce due to the lack of radiative lifetime measurements. The existing results are mostly concerning the low excitation levels. Concretely, in 1983 Hannaford and Lowe [7] measured lifetimes for 15 levels of the $z^{6} \mathrm{~F}^{\circ}, z^{4} \mathrm{P}^{\circ}, z^{6} \mathrm{P}^{\circ}$ and $z^{6} \mathrm{D}^{\circ}$ terms using the LIF method applied to a sputtered metal vapour. Later on, Sikström et al. [8] reported experimental radiative lifetimes for ten levels by the LIF technique. With a HFR approximation including core polarization effects, theoretical lifetimes for 37 levels of Mo II and the oscillator

\footnotetext{
a e-mail: e.biemont@ulg.ac.be

b e-mail: dai@jlu.edu.cn
}

strengths of the depopulating transitions were calculated by Quinet [9]. Very recently, Lundberg et al. [10] measured new radiative lifetimes for 14 odd levels in the energy range $48000-61000 \mathrm{~cm}^{-1}$ with the method of LIF spectroscopy. To our best knowledge, however, no experimental radiative lifetimes have been reported in the literature for the levels above $61000 \mathrm{~cm}^{-1}$ although transitions from these levels are likely to be observed in astrophysics and in plasma physics.

In the present paper, the lifetimes for 13 Mo II levels were measured by the TR-LIF method and, among them, ten levels are located above $61000 \mathrm{~cm}^{-1}$. The lifetimes of the levels at 48022.82 and $60992.76 \mathrm{~cm}^{-1}$, which were investigated previously by Hannaford and Lowe [7], Sikström et al. [8] and by Lundberg et al. [10], respectively, were also measured in the present paper for comparison. Corresponding theoretical lifetime values were also calculated using the multiconfigurational HFR method taking core-polarization effects into account, and they are in good agreement with the experimental results having in mind the complexity of the calculations in such heavy atomic systems. In addition, new theoretical transition probabilities and oscillator strengths for a set of 246 transitions in the wavelength range from 207.431 to $510.813 \mathrm{~nm}$ were also obtained using the HFR method.

\section{Lifetime measurements}

The experimental setup used for the lifetime measurements is similar to the one described in our paper [11], and 


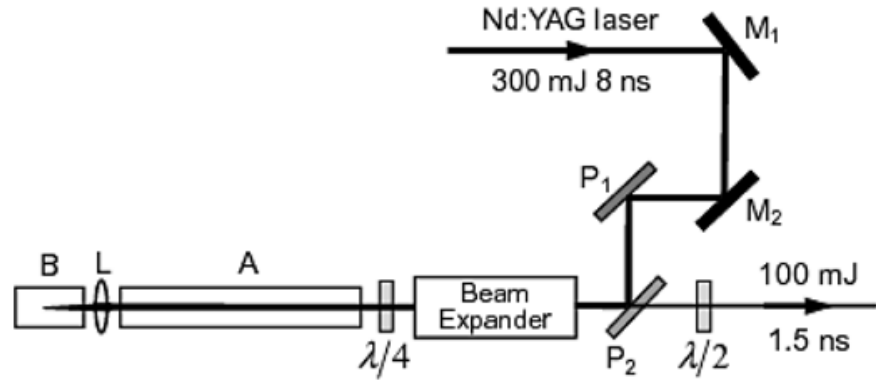

Fig. 1. Stimulated Brillouin scattering (SBS) compressor. $\lambda / 2$ (half-wave) and $\lambda / 4$ (quarter-wave) plates are inserted. $P_{1}$ and $P_{2}$ are plate polarizers placed at the Brewster angle. $\mathrm{M}_{1}$ and $\mathrm{M}_{2}$ are $532 \mathrm{~nm}$ totally reflecting mirrors. $\mathrm{L}$ is a lens with a focal length of $12.5 \mathrm{~cm}$. A is a $150 \mathrm{~cm}$ and B a $30 \mathrm{~cm}$ polyvinylchloride (PVC) tube full of pure water.

only a brief outline is given here. Two Nd:YAG lasers both having the output wavelengths of $532 \mathrm{~nm}$, pulse durations of about $8 \mathrm{~ns}$, and repetition rates of $10 \mathrm{~Hz}$ were used in the experiment. One of them was focused on a rotating molybdenum foil to produce laser-induced Mo plasma. The pulse duration of the other one was compressed to 1-2 ns by a stimulated Brillouin scattering (SBS) compressor. The setup of the pulse compressor is illustrated in Figure 1. A detailed description of this technique has been presented by Schiemann et al. [12]. The compressed pulses were conducted into a dye laser (Sirah Cobra-Stretch) with a $0.08 \mathrm{~cm}^{-1}$ linewidth operated with a DCM dye. The third harmonic of the dye laser, generated by two $\mathrm{BBO}$ crystals and a retarding plate, was horizontally sent into the vacuum chamber to excite Mo II ions. The delay time between the excitation and ablation pulses can be tuned by a pulse delay generator (SRS DG535). Fluorescence from a measured level was dispersed by a grating monochromator and then detected by a microchannel plate photomultiplier tube (Hamamatsu R3809U-58) with a 163 -ps rise time. A $2.5 \mathrm{GHz}$ digital oscilloscope (Tektronix DPO 7254) was used to register and average the transient signals from the detector. Each fluorescence decay curve was obtained by averaging over 1000 shots to obtain a good signal-to-noise ratio, and more than 10 curves under different conditions were recorded for each level to get the final lifetime.

The partial diagram of the energy levels and the excitation schemes followed in the present experiment are shown in Figure 2. The energy level data were taken from the paper of Nilsson and Pickering [13]. In the measurements, due to the finite linewidth of the dye laser, the exciting wavelength has been carefully chosen from all available excitation pathways to avoid simultaneous excitation of other levels. Moreover, careful attention has been paid to the effects such as the radiation trapping effect, the collisional effect, the flight-out-of-view effect, and the saturation effect which could possibly contribute to the systematic errors of the experimental results. The details for checking and eliminating these effects were described in our paper [11]. The lifetime values were performed by fitting the experimental fluorescence decay curve to

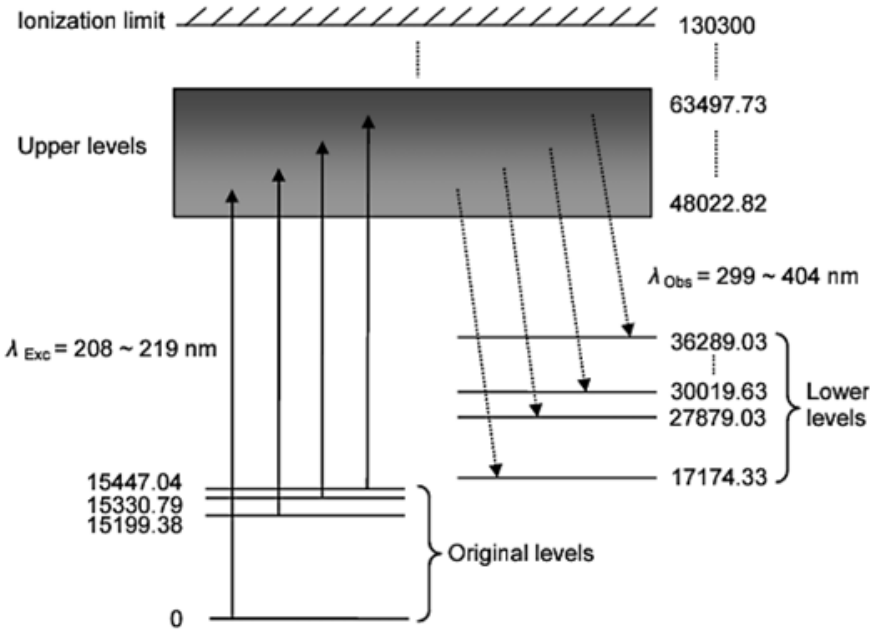

Fig. 2. Partial energy-level diagram of Mo II and excitation schemes. The solid arrows indicate the excitation pathways and the dashed arrows indicate the observation channels. The energy levels are expressed in $\mathrm{cm}^{-1}$.

a convolution of the recorded laser excitation pulse and a pure exponential function. A typical fluorescence decay curve for the $63298.54 \mathrm{~cm}^{-1}$ level together with an exciting pulse and a fitted convolution curve is shown in Figure 3.

\section{Theoretical calculations}

Lifetimes, transition probabilities and branching fractions were calculated using the relativistic HFR approach [14] including core-polarization corrections (see e.g. [15]). As the physical model considered was exactly the same as the one described in our recent study of Mo II [10], the details will not be repeated here. Let us just remind that, in the latter work, the configurations $4 d^{5}, 4 d^{4} 5 s, 4 d^{4} 6 s, 4 d^{4} 5 d$, $4 d^{3} 5 s^{2}, 4 d^{3} 5 p^{2}, 4 d^{3} 5 d^{2}, 4 d^{3} 5 s 5 d$ (even parity) and $4 d^{4} 5 p$, $4 d^{4} 6 p, 4 d^{4} 4 f, 4 d^{4} 5 f, 4 d^{3} 5 s 5 p, 4 d^{3} 5 p 5 d$ (odd parity) were included in the calculations with the dipole polarizability $\alpha_{d}=5.67 a_{0}^{3}$ and the cut off radius $r_{c}=1.73 a_{0}$ as corepolarization parameters. This model was then combined with a semi-empirical adjustment of the radial parameters using the experimental energy levels published by Nilsson and Pickering [13] which allowed us to refine the study previously performed by Quinet [9].

\section{Results and discussion}

In the present paper 13 lifetimes for highly excited oddparity Mo II levels from 48000 to $63500 \mathrm{~cm}^{-1}$ belonging to the $4 d^{4} 5 p$ configuration have been measured and presented in Table 1 together with the HFR theoretical lifetimes. The results fall in the range from 2.2 to $7.5 \mathrm{~ns}$. One can see that the lifetime of the $48022.82 \mathrm{~cm}^{-1}$ level reported by Hannaford and Lowe [7] and Sikström et al. [8] with the LIF method agrees well with our result. But for 
Table 1. Measured lifetimes of 13 odd-parity $4 d^{4} 5 p$ levels in Mo II.

\begin{tabular}{|c|c|c|c|c|c|c|c|c|}
\hline \multicolumn{4}{|c|}{ Upper level $^{\mathrm{a}}$} & \multirow[b]{2}{*}{$\begin{array}{l}\lambda_{\text {Exc. }} \\
(\mathrm{nm})\end{array}$} & \multirow[b]{2}{*}{$\begin{array}{l}\lambda_{\text {Obs. }} \\
(\mathrm{nm})\end{array}$} & \multicolumn{3}{|c|}{ Lifetime (ns) } \\
\hline Config. & Term & $J$ & $E\left(\mathrm{~cm}^{-1}\right)$ & & & $\begin{array}{c}\text { This work } \\
\text { Expt. }\end{array}$ & $\begin{array}{c}\text { This work } \\
\text { HFR }\end{array}$ & $\begin{array}{c}\text { Previous } \\
\text { Expt. }\end{array}$ \\
\hline $4 d^{4}\left({ }^{5} \mathrm{D}\right) 5 p$ & $z^{4} \mathrm{P}^{\circ}$ & $3 / 2$ & 48022.82 & 208.234 & 324 & $4.8(2)$ & 4.8 & $4.8(4)^{\mathrm{b}}, 4.9(3)^{\mathrm{c}}$ \\
\hline $4 d^{4}\left({ }^{3} \mathrm{H}\right) 5 p$ & $z^{2} \mathrm{G}^{\circ}$ & $9 / 2$ & 60973.46 & 219.093 & 320 & $4.8(4)$ & 5.6 & \\
\hline $4 d^{4}\left(\mathrm{a}^{3} \mathrm{~F}\right) 5 p$ & $z^{2} \mathrm{D}^{\circ}$ & $5 / 2$ & 60992.76 & 219.006 & 404 & $7.5(5)$ & 7.5 & $6.6^{\mathrm{d}}$ \\
\hline $4 d^{4}\left(\mathrm{a}^{3} \mathrm{P}\right) 5 p$ & $z^{2} \mathrm{P}^{\circ}$ & $3 / 2$ & 61746.93 & 214.834 & 356 & $5.1(2)$ & 5.0 & \\
\hline $4 d^{4}\left(\mathrm{a}^{3} \mathrm{P}\right) 5 p$ & $y{ }^{4} \mathrm{P}^{\circ}$ & $5 / 2$ & 62425.75 & 211.746 & 295 & $3.0(2)$ & 3.1 & \\
\hline $4 d^{4}\left(\mathrm{a}^{3} \mathrm{~F}\right) 5 p$ & $x^{4} \mathrm{D}^{\circ}$ & $7 / 2$ & 62492.15 & 211.449 & 297 & $3.1(2)$ & 3.9 & \\
\hline $4 d^{4}\left({ }^{3} \mathrm{H}\right) 5 p$ & $z^{2} \mathrm{I}^{\circ}$ & $11 / 2$ & 62728.65 & 211.499 & 350 & $4.7(4)$ & 4.2 & \\
\hline $4 d^{4}\left(\mathrm{a}^{3} \mathrm{~F}\right) 5 p$ & $y{ }^{4} \mathrm{~F}^{\circ}$ & $3 / 2$ & 63002.97 & 211.401 & 329 & $2.7(2)$ & 2.6 & \\
\hline $4 d^{4}\left({ }^{3} \mathrm{H}\right) 5 p$ & $y^{4} \mathrm{G}^{\circ}$ & $9 / 2$ & 63012.56 & 209.724 & 310 & $2.2(1)$ & 2.7 & \\
\hline $4 d^{4}\left({ }^{3} \mathrm{H}\right) 5 p$ & $y^{4} \mathrm{G}^{\circ}$ & $5 / 2$ & 63041.81 & 209.019 & 308 & $2.2(1)$ & 2.4 & \\
\hline $4 d^{4}\left({ }^{3} \mathrm{G}\right) 5 p$ & $y{ }^{4} \mathrm{H}^{\circ}$ & $7 / 2$ & 63298.54 & 208.896 & 299 & $3.6(2)$ & 3.5 & \\
\hline $4 d^{4}\left(\mathrm{a}^{3} \mathrm{~F}\right) 5 p$ & $x^{4} \mathrm{D}^{\circ}$ & $5 / 2$ & 63392.75 & 208.065 & 294 & $2.9(1)$ & 3.1 & \\
\hline $4 d^{4}\left({ }^{3} \mathrm{H}\right) 5 p$ & $z^{2} \mathrm{H}^{\circ}$ & $9 / 2$ & 63497.73 & 208.114 & 319 & $3.8(1)$ & 3.5 & \\
\hline
\end{tabular}

${ }^{\mathrm{a}}$ Nilsson and Pickering [13]. ${ }^{\mathrm{b}}$ Hannaford and Lowe [7]. ${ }^{\mathrm{c}}$ Sikström et al. [8]. ${ }^{\mathrm{d}}$ Lundberg et al. [10].

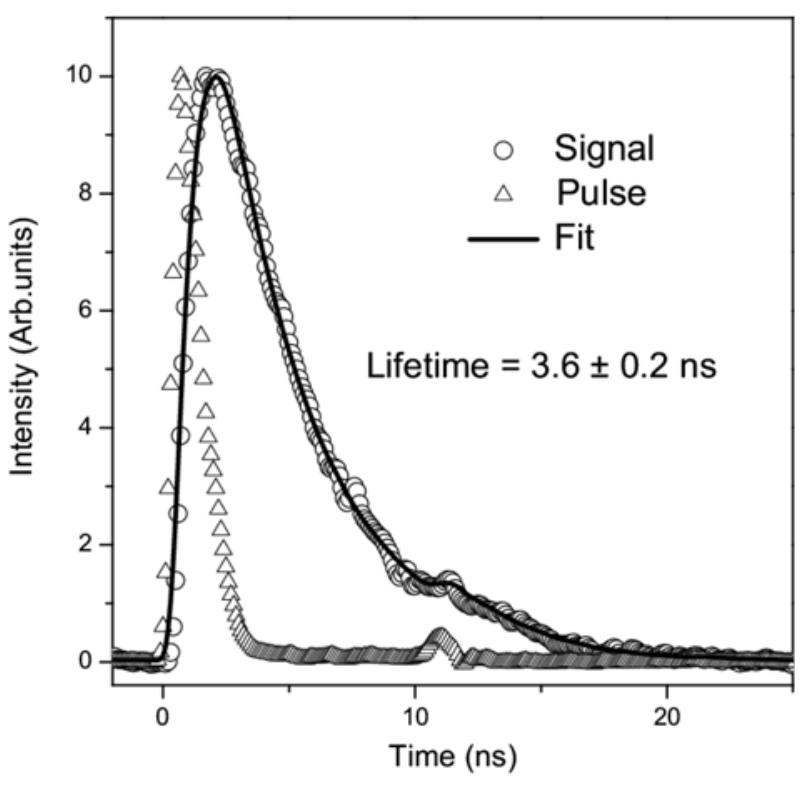

Fig. 3. A decay curve of the $63298.54 \mathrm{~cm}^{-1}$ level together with the fitted convolution curve between the laser pulse and an exponential with a lifetime of $3.6 \mathrm{~ns}$.

the $60992.76 \mathrm{~cm}^{-1}$ level, there is a difference reaching $13.6 \%$ between the result by Lundberg et al. [10] and ours (using the former as the reference). There is no clear explanation for this discrepancy. As it can be seen from Table 1 however, our result is much closer to the theoretical HFR value.

The differences between the HFR and experimental lifetimes are within $\pm 10 \%$ (using the experimental values as the references) except for the levels $60973.46,62492.15$, 62728.65 and $63012.56 \mathrm{~cm}^{-1}$ of which the discrepancies are $16.7 \%, 25.8 \%, 10.6 \%$ and $22.7 \%$, respectively. It may be concluded that the HFR theoretical results agree basi- cally well with the experimental lifetimes measured in this paper.

In Table 2, the transition probabilities $\left(A_{k i}\right.$ in $\left.10^{7} \mathrm{~s}^{-1}\right)$ and oscillator strengths (log $g f$ ) are presented for 246 transitions depopulating the levels for which the lifetimes have been measured in the present work. Besides the HFR results, the corrected values which were obtained from a combination of the experimental lifetimes and the HFR branching fractions are also presented for comparison.

\section{Conclusions}

The lifetimes of 13 highly excited odd-parity levels of Mo II in the range of $48000-63500 \mathrm{~cm}^{-1}$ have been measured using the TR-LIF technique in a laser plasma. To our best knowledge, 11 lifetimes among them are reported for the first time. The results are from 2.2 to $7.5 \mathrm{~ns}$ with the uncertainties within $\pm 10 \%$. A comparison was performed between the experimental lifetimes and theoretical HFR values, and a rather good agreement is achieved. Moreover, the HFR transition probabilities and oscillator strengths of the lines depopulating these levels were calculated and compared with the corrected ones which were obtained by combining the measured lifetimes with the HFR branching fractions. We believe that these results are more useful in some fields such as plasma physics, and astronomical spectroscopy etc.

This work was supported by the National Natural Science Foundation of China (Grant Nos. 10974066 and 10978012) and by the Program for New Century Excellent Talents in University of the Ministry of Education of China (Grant No. NCET-05-0302). Financial support from the Belgian FRSFNRS is also acknowledged. 
Table 2. Transition probabilities $(g A)$ and oscillator strengths (log $g f$ ) for Mo II transitions depopulating odd-parity levels. Only transitions below $1000.0 \mathrm{~nm}$ with $g A$-values greater than $10^{7} \mathrm{~s}^{-1}$ are listed.

\begin{tabular}{|c|c|c|c|c|c|c|c|c|}
\hline \multirow{2}{*}{$\begin{array}{c}\text { Upper level }^{\mathrm{a}} \\
\left(\mathrm{cm}^{-1}\right)\end{array}$} & \multirow{2}{*}{$J$} & \multirow{2}{*}{$\begin{array}{c}\text { Lower level }^{\mathrm{a}} \\
\left(\mathrm{cm}^{-1}\right)\end{array}$} & \multirow{2}{*}{$J$} & \multirow{2}{*}{$\begin{array}{c}\lambda_{\text {air }} \\
(\mathrm{nm})\end{array}$} & \multicolumn{2}{|c|}{$g A\left(10^{7} \mathrm{~s}^{-1}\right)$} & \multicolumn{2}{|c|}{$\log (g f)$} \\
\hline & & & & & HFR & Corr. $^{b}$ & HFR & Corr. $^{b}$ \\
\hline \multirow[t]{12}{*}{48022.82} & $3 / 2$ & 0 & $5 / 2$ & 208.168 & 11.00 & 11.00 & -1.15 & -1.15 \\
\hline & & 11783.65 & $1 / 2$ & 275.863 & 1.91 & 1.91 & -1.66 & -1.66 \\
\hline & & 12034.42 & $3 / 2$ & 277.785 & 1.78 & 1.78 & -1.69 & -1.69 \\
\hline & & 12417.63 & $5 / 2$ & 280.775 & 31.30 & 31.30 & -0.43 & -0.43 \\
\hline & & 15691.45 & $5 / 2$ & 309.207 & 12.50 & 12.50 & -0.75 & -0.75 \\
\hline & & 15890.42 & $1 / 2$ & 311.122 & 1.33 & 1.33 & -1.71 & -1.71 \\
\hline & & 16796.47 & $1 / 2$ & 320.150 & 2.97 & 2.97 & -1.34 & -1.34 \\
\hline & & 17174.33 & $3 / 2$ & 324.072 & 7.13 & 7.13 & -0.95 & -0.95 \\
\hline & & 17344.36 & $5 / 2$ & 325.868 & 2.49 & 2.49 & -1.40 & -1.40 \\
\hline & & 24659.50 & $3 / 2$ & 427.901 & 3.67 & 3.67 & -1.00 & -1.00 \\
\hline & & 24836.32 & $5 / 2$ & 431.164 & 1.11 & 1.11 & -1.51 & -1.51 \\
\hline & & 25112.57 & $5 / 2$ & 436.363 & 3.87 & 3.87 & -0.96 & -0.96 \\
\hline \multirow[t]{26}{*}{60973.46} & $9 / 2$ & 15330.79 & $7 / 2$ & 219.025 & 6.86 & 8.00 & -1.31 & -1.24 \\
\hline & & 15427.90 & $9 / 2$ & 219.492 & 1.32 & 1.54 & -2.02 & -1.95 \\
\hline & & 15447.04 & $11 / 2$ & 219.584 & 1.02 & 1.19 & -2.13 & -2.06 \\
\hline & & 16947.08 & $7 / 2$ & 227.066 & 1.13 & 1.32 & -2.06 & -1.99 \\
\hline & & 23833.15 & $9 / 2$ & 269.169 & 3.29 & 3.84 & -1.44 & -1.37 \\
\hline & & 23853.58 & $7 / 2$ & 269.317 & 14.10 & 16.45 & -0.81 & -0.74 \\
\hline & & 24509.50 & $7 / 2$ & 274.162 & 24.00 & 28.00 & -0.57 & -0.50 \\
\hline & & 26041.36 & $7 / 2$ & 286.186 & 5.49 & 6.41 & -1.17 & -1.10 \\
\hline & & 26068.81 & $9 / 2$ & 286.411 & 5.08 & 5.93 & -1.20 & -1.13 \\
\hline & & 26405.84 & $7 / 2$ & 289.203 & 6.33 & 7.39 & -1.10 & -1.03 \\
\hline & & 26488.47 & $9 / 2$ & 289.896 & 1.81 & 2.11 & -1.64 & -1.57 \\
\hline & & 26739.74 & $11 / 2$ & 292.024 & 6.69 & 7.81 & -1.07 & -1.00 \\
\hline & & 27410.47 & $7 / 2$ & 297.860 & 19.40 & 22.63 & -0.59 & -0.52 \\
\hline & & 27627.03 & $11 / 2$ & 299.795 & 5.70 & 6.65 & -1.11 & -1.04 \\
\hline & & 27724.78 & $9 / 2$ & 300.676 & 1.38 & 1.61 & -1.73 & -1.66 \\
\hline & & 28989.22 & $7 / 2$ & 312.563 & 5.33 & 6.22 & -1.11 & -1.04 \\
\hline & & 29034.43 & $9 / 2$ & 313.006 & 14.50 & 16.92 & -0.67 & -0.60 \\
\hline & & 30019.63 & $7 / 2$ & 322.969 & 25.40 & 29.63 & -0.40 & -0.33 \\
\hline & & 30213.84 & $9 / 2$ & 325.008 & 2.79 & 3.26 & -1.35 & -1.28 \\
\hline & & 30391.61 & $11 / 2$ & 326.897 & 5.31 & 6.20 & -1.07 & -1.00 \\
\hline & & 33146.56 & $7 / 2$ & 359.262 & 1.52 & 1.77 & -1.53 & -1.46 \\
\hline & & 33254.67 & $9 / 2$ & 360.663 & 2.91 & 3.40 & -1.24 & -1.17 \\
\hline & & 33601.41 & $11 / 2$ & 365.232 & 10.40 & 12.13 & -0.68 & -0.61 \\
\hline & & 36741.56 & $7 / 2$ & 412.563 & 2.09 & 2.44 & -1.27 & -1.20 \\
\hline & & 37431.72 & $7 / 2$ & 424.658 & 1.52 & 1.77 & -1.39 & -1.32 \\
\hline & & 38054.12 & $9 / 2$ & 436.190 & 1.42 & 1.66 & -1.39 & -1.32 \\
\hline \multirow[t]{17}{*}{60992.76} & $5 / 2$ & 15330.79 & $7 / 2$ & 218.932 & 4.53 & 4.53 & -1.49 & -1.49 \\
\hline & & 15699.39 & $3 / 2$ & 220.714 & 2.04 & 2.04 & -1.82 & -1.82 \\
\hline & & 16947.08 & $7 / 2$ & 226.967 & 5.55 & 5.55 & -1.37 & -1.37 \\
\hline & & 22444.51 & $5 / 2$ & 259.338 & 13.40 & 13.40 & -0.87 & -0.87 \\
\hline & & 24137.91 & $3 / 2$ & 271.254 & 1.41 & 1.41 & -1.81 & -1.81 \\
\hline & & 25112.57 & $5 / 2$ & 278.623 & 1.53 & 1.53 & -1.75 & -1.75 \\
\hline & & 27410.47 & $7 / 2$ & 297.689 & 6.31 & 6.31 & -1.08 & -1.08 \\
\hline & & 27627.86 & $3 / 2$ & 299.629 & 7.01 & 7.01 & -1.03 & -1.03 \\
\hline & & 28884.05 & $3 / 2$ & 311.352 & 1.97 & 1.97 & -1.54 & -1.54 \\
\hline & & 33086.48 & $3 / 2$ & 358.240 & 2.05 & 2.05 & -1.40 & -1.40 \\
\hline & & 33146.56 & $7 / 2$ & 359.013 & 3.11 & 3.11 & -1.22 & -1.22 \\
\hline & & 33525.42 & $3 / 2$ & 363.965 & 2.98 & 2.98 & -1.22 & -1.22 \\
\hline & & 34419.52 & $3 / 2$ & 376.211 & 3.13 & 3.13 & -1.17 & -1.17 \\
\hline & & 36741.56 & $7 / 2$ & 412.234 & 11.00 & 11.00 & -0.55 & -0.55 \\
\hline & & 37431.72 & $7 / 2$ & 424.310 & 2.38 & 2.38 & -1.19 & -1.19 \\
\hline & & 39913.21 & $5 / 2$ & 474.261 & 2.76 & 2.76 & -1.03 & -1.03 \\
\hline & & 41421.58 & $5 / 2$ & 510.813 & 1.02 & 1.02 & -1.40 & -1.40 \\
\hline
\end{tabular}


Table 2. continued.

\begin{tabular}{|c|c|c|c|c|c|c|c|c|}
\hline \multirow{2}{*}{$\begin{array}{c}\text { Upper level }^{\mathrm{a}} \\
\left(\mathrm{cm}^{-1}\right)\end{array}$} & \multirow{2}{*}{$J$} & \multirow{2}{*}{$\begin{array}{c}\text { Lower level }^{\mathrm{a}} \\
\left(\mathrm{cm}^{-1}\right)\end{array}$} & \multirow{2}{*}{$J$} & \multirow{2}{*}{$\begin{array}{c}\lambda_{\text {air }} \\
(\mathrm{nm})\end{array}$} & \multicolumn{2}{|c|}{$g A\left(10^{7} \mathrm{~s}^{-1}\right)$} & \multicolumn{2}{|c|}{$\log (g f)$} \\
\hline & & & & & HFR & Corr. $^{b}$ & HFR & Corr. $^{b}$ \\
\hline \multirow[t]{17}{*}{61746.93} & $3 / 2$ & 15199.38 & $5 / 2$ & 214.767 & 1.42 & 1.39 & -2.00 & -2.01 \\
\hline & & 15691.45 & $5 / 2$ & 217.061 & 2.52 & 2.47 & -1.75 & -1.76 \\
\hline & & 15890.42 & $1 / 2$ & 218.003 & 5.78 & 5.67 & -1.38 & -1.39 \\
\hline & & 17174.33 & $3 / 2$ & 224.284 & 4.24 & 4.16 & -1.49 & -1.50 \\
\hline & & 17344.36 & $5 / 2$ & 225.142 & 8.59 & 8.42 & -1.18 & -1.19 \\
\hline & & 22444.51 & $5 / 2$ & 254.361 & 5.84 & 5.73 & -1.24 & -1.25 \\
\hline & & 23934.63 & $5 / 2$ & 264.386 & 3.54 & 3.47 & -1.42 & -1.43 \\
\hline & & 24836.32 & $5 / 2$ & 270.845 & 1.42 & 1.39 & -1.80 & -1.81 \\
\hline & & 26603.86 & $1 / 2$ & 284.468 & 2.41 & 2.36 & -1.53 & -1.54 \\
\hline & & 27627.86 & $3 / 2$ & 293.006 & 1.15 & 1.13 & -1.83 & -1.84 \\
\hline & & 28884.05 & $3 / 2$ & 304.206 & 1.15 & 1.13 & -1.79 & -1.80 \\
\hline & & 29022.46 & $5 / 2$ & 305.493 & 10.90 & 10.69 & -0.82 & -0.83 \\
\hline & & 32124.34 & $1 / 2$ & 337.483 & 2.26 & 2.22 & -1.40 & -1.41 \\
\hline & & 32879.90 & $5 / 2$ & 346.317 & 2.34 & 2.29 & -1.37 & -1.38 \\
\hline & & 33086.48 & $3 / 2$ & 348.813 & 3.44 & 3.37 & -1.20 & -1.21 \\
\hline & & 33525.42 & $3 / 2$ & 354.239 & 5.46 & 5.35 & -0.98 & -0.99 \\
\hline & & 34419.52 & $3 / 2$ & 365.829 & 10.70 & 10.49 & -0.66 & -0.67 \\
\hline \multirow[t]{25}{*}{62425.75} & $5 / 2$ & 15199.38 & $5 / 2$ & 211.679 & 41.20 & 42.57 & -0.56 & -0.54 \\
\hline & & 15330.79 & $7 / 2$ & 212.270 & 2.71 & 2.80 & -1.74 & -1.72 \\
\hline & & 15699.39 & $3 / 2$ & 213.945 & 11.10 & 11.47 & -1.12 & -1.10 \\
\hline & & 16947.08 & $7 / 2$ & 219.815 & 18.90 & 19.53 & -0.86 & -0.84 \\
\hline & & 17344.36 & $5 / 2$ & 221.752 & 6.82 & 7.05 & -1.30 & -1.28 \\
\hline & & 22444.51 & $5 / 2$ & 250.042 & 10.60 & 10.95 & -1.00 & -0.98 \\
\hline & & 22864.47 & $3 / 2$ & 252.696 & 1.23 & 1.27 & -1.93 & -1.91 \\
\hline & & 23853.58 & $7 / 2$ & 259.177 & 3.71 & 3.83 & -1.43 & -1.41 \\
\hline & & 24137.91 & $3 / 2$ & 261.102 & 1.19 & 1.23 & -1.91 & -1.89 \\
\hline & & 24509.50 & $7 / 2$ & 263.661 & 5.26 & 5.44 & -1.26 & -1.24 \\
\hline & & 24659.50 & $3 / 2$ & 264.708 & 2.13 & 2.20 & -1.65 & -1.63 \\
\hline & & 25341.90 & $7 / 2$ & 269.579 & 2.23 & 2.30 & -1.61 & -1.59 \\
\hline & & 26041.36 & $7 / 2$ & 274.762 & 4.54 & 4.69 & -1.29 & -1.27 \\
\hline & & 26405.84 & $7 / 2$ & 277.542 & 21.30 & 22.01 & -0.61 & -0.59 \\
\hline & & 27627.86 & $3 / 2$ & 287.289 & 10.20 & 10.54 & -0.90 & -0.88 \\
\hline & & 27879.03 & $5 / 2$ & 289.378 & 3.20 & 3.31 & -1.40 & -1.38 \\
\hline & & 28884.05 & $3 / 2$ & 298.049 & 3.98 & 4.11 & -1.28 & -1.26 \\
\hline & & 28989.22 & $7 / 2$ & 298.987 & 1.74 & 1.80 & -1.63 & -1.61 \\
\hline & & 29022.46 & $5 / 2$ & 299.285 & 18.50 & 19.12 & -0.61 & -0.59 \\
\hline & & 29699.47 & $5 / 2$ & 305.476 & 5.27 & 5.45 & -1.13 & -1.11 \\
\hline & & 33146.56 & $7 / 2$ & 341.442 & 4.37 & 4.52 & -1.11 & -1.09 \\
\hline & & 33525.42 & $3 / 2$ & 345.918 & 1.02 & 1.05 & -1.73 & -1.71 \\
\hline & & 33549.54 & $7 / 2$ & 346.207 & 2.46 & 2.54 & -1.35 & -1.33 \\
\hline & & 37431.72 & $7 / 2$ & 399.983 & 2.95 & 3.05 & -1.15 & -1.13 \\
\hline & & 39243.69 & $3 / 2$ & 431.247 & 1.41 & 1.46 & -1.40 & -1.38 \\
\hline \multirow[t]{17}{*}{62492.15} & $7 / 2$ & 15199.38 & $5 / 2$ & 211.382 & 2.62 & 3.30 & -1.75 & -1.65 \\
\hline & & 15330.79 & $7 / 2$ & 211.971 & 17.10 & 21.51 & -0.94 & -0.84 \\
\hline & & 15427.90 & $9 / 2$ & 212.408 & 4.54 & 5.71 & -1.51 & -1.41 \\
\hline & & 15691.45 & $5 / 2$ & 213.605 & 20.50 & 25.79 & -0.85 & -0.75 \\
\hline & & 16947.08 & $7 / 2$ & 219.494 & 5.82 & 7.32 & -1.37 & -1.27 \\
\hline & & 17344.36 & $5 / 2$ & 221.426 & 18.90 & 23.78 & -0.86 & -0.76 \\
\hline & & 22444.51 & $5 / 2$ & 249.627 & 4.84 & 6.09 & -1.34 & -1.24 \\
\hline & & 23833.15 & $9 / 2$ & 258.595 & 29.90 & 37.62 & -0.52 & -0.42 \\
\hline & & 23934.63 & $5 / 2$ & 259.275 & 4.38 & 5.51 & -1.35 & -1.25 \\
\hline & & 24836.32 & $5 / 2$ & 265.484 & 5.25 & 6.60 & -1.25 & -1.15 \\
\hline & & 25341.90 & $7 / 2$ & 269.097 & 2.28 & 2.87 & -1.61 & -1.51 \\
\hline & & 26405.84 & $7 / 2$ & 277.032 & 1.94 & 2.44 & -1.65 & -1.55 \\
\hline & & 26488.47 & $9 / 2$ & 277.668 & 17.60 & 22.14 & -0.69 & -0.59 \\
\hline & & 28877.24 & $5 / 2$ & 297.400 & 1.86 & 2.34 & -1.61 & -1.51 \\
\hline & & 28989.22 & $7 / 2$ & 298.394 & 2.27 & 2.86 & -1.52 & -1.42 \\
\hline & & 29022.46 & $5 / 2$ & 298.691 & 28.80 & 36.23 & -0.41 & -0.31 \\
\hline & & 29034.43 & $9 / 2$ & 298.798 & 21.10 & 26.55 & -0.55 & -0.45 \\
\hline
\end{tabular}


Table 2. continued.

\begin{tabular}{|c|c|c|c|c|c|c|c|c|}
\hline \multirow{2}{*}{$\begin{array}{c}\text { Upper level }^{\mathrm{a}} \\
\left(\mathrm{cm}^{-1}\right)\end{array}$} & \multirow{2}{*}{$J$} & \multirow{2}{*}{$\begin{array}{c}\text { Lower level }^{\mathrm{a}} \\
\left(\mathrm{cm}^{-1}\right)\end{array}$} & \multirow{2}{*}{$J$} & \multirow{2}{*}{$\begin{array}{l}\lambda_{\text {air }} \\
(\mathrm{nm})\end{array}$} & \multicolumn{2}{|c|}{$g A\left(10^{7} \mathrm{~s}^{-1}\right)$} & \multicolumn{2}{|c|}{$\log (g f)$} \\
\hline & & & & & HFR & Corr. $^{b}$ & HFR & Corr. $^{b}$ \\
\hline \multirow{18}{*}{62728.65} & \multirow{18}{*}{$11 / 2$} & 30019.63 & $7 / 2$ & 307.863 & 4.52 & 5.69 & -1.19 & -1.09 \\
\hline & & 36289.03 & $5 / 2$ & 381.526 & 1.21 & 1.52 & -1.58 & -1.48 \\
\hline & & 36741.56 & $7 / 2$ & 388.231 & 2.41 & 3.03 & -1.26 & -1.16 \\
\hline & & 15427.90 & $9 / 2$ & 211.346 & 2.97 & 2.65 & -1.70 & -1.75 \\
\hline & & 15447.04 & $11 / 2$ & 211.432 & 41.20 & 36.82 & -0.56 & -0.61 \\
\hline & & 22980.45 & $11 / 2$ & 251.508 & 14.50 & 12.96 & -0.86 & -0.91 \\
\hline & & 26068.81 & $9 / 2$ & 272.697 & 50.20 & 44.86 & -0.25 & -0.30 \\
\hline & & 26488.47 & $9 / 2$ & 275.855 & 1.80 & 1.61 & -1.69 & -1.74 \\
\hline & & 27114.16 & $13 / 2$ & 280.702 & 20.40 & 18.23 & -0.62 & -0.67 \\
\hline & & 27724.78 & $9 / 2$ & 285.599 & 27.90 & 24.93 & -0.47 & -0.52 \\
\hline & & 29034.43 & $9 / 2$ & 296.700 & 3.47 & 3.10 & -1.34 & -1.39 \\
\hline & & 30213.84 & $9 / 2$ & 307.463 & 5.63 & 5.03 & -1.10 & -1.15 \\
\hline & & 30391.61 & $11 / 2$ & 309.153 & 2.81 & 2.51 & -1.40 & -1.45 \\
\hline & & 33045.60 & $9 / 2$ & 336.796 & 80.50 & 71.94 & 0.14 & 0.09 \\
\hline & & 33254.67 & $9 / 2$ & 339.185 & 9.38 & 8.38 & -0.79 & -0.84 \\
\hline & & 33601.41 & $11 / 2$ & 343.223 & 8.32 & 7.43 & -0.83 & -0.88 \\
\hline & & 35099.69 & $13 / 2$ & 361.836 & 2.09 & 1.87 & -1.39 & -1.44 \\
\hline & & 35406.26 & $11 / 2$ & 365.896 & 10.30 & 9.20 & -0.69 & -0.74 \\
\hline \multirow[t]{12}{*}{63002.97} & \multirow[t]{12}{*}{$3 / 2$} & 15199.38 & $5 / 2$ & 209.123 & 41.80 & 40.25 & -0.56 & -0.58 \\
\hline & & 15691.45 & $5 / 2$ & 211.298 & 2.30 & 2.21 & -1.81 & -1.83 \\
\hline & & 15890.42 & $1 / 2$ & 212.191 & 2.87 & 2.76 & -1.71 & -1.73 \\
\hline & & 16796.47 & $1 / 2$ & 216.352 & 4.78 & 4.60 & -1.47 & -1.49 \\
\hline & & 17174.33 & $3 / 2$ & 218.136 & 17.90 & 17.24 & -0.89 & -0.91 \\
\hline & & 17344.36 & $5 / 2$ & 218.948 & 5.72 & 5.51 & -1.39 & -1.41 \\
\hline & & 22864.47 & $3 / 2$ & 249.062 & 2.66 & 2.56 & -1.61 & -1.63 \\
\hline & & 23934.63 & $5 / 2$ & 255.885 & 3.06 & 2.95 & -1.52 & -1.54 \\
\hline & & 24137.91 & $3 / 2$ & 257.224 & 15.10 & 14.54 & -0.82 & -0.84 \\
\hline & & 24372.41 & $1 / 2$ & 258.785 & 3.95 & 3.80 & -1.40 & -1.42 \\
\hline & & 27627.86 & $3 / 2$ & 282.602 & 1.46 & 1.41 & -1.76 & -1.78 \\
\hline & & 28884.05 & $3 / 2$ & 293.007 & 44.60 & 42.95 & -0.24 & -0.26 \\
\hline \multirow{20}{*}{63012.56} & \multirow{20}{*}{$9 / 2$} & 15427.90 & $9 / 2$ & 210.085 & 10.20 & 12.52 & -1.17 & -1.08 \\
\hline & & 15447.04 & $11 / 2$ & 210.170 & 129.00 & 158.32 & -0.07 & 0.02 \\
\hline & & 16947.08 & $7 / 2$ & 217.014 & 27.00 & 33.14 & -0.72 & -0.63 \\
\hline & & 22980.45 & $11 / 2$ & 249.724 & 2.30 & 2.82 & -1.67 & -1.58 \\
\hline & & 23833.15 & $9 / 2$ & 255.160 & 17.00 & 20.86 & -0.78 & -0.69 \\
\hline & & 23853.58 & $7 / 2$ & 255.293 & 4.65 & 5.71 & -1.34 & -1.25 \\
\hline & & 24509.50 & $7 / 2$ & 259.642 & 3.05 & 3.74 & -1.51 & -1.42 \\
\hline & & 25341.90 & $7 / 2$ & 265.380 & 12.90 & 15.83 & -0.87 & -0.78 \\
\hline & & 26405.84 & $7 / 2$ & 273.093 & 11.40 & 13.99 & -0.90 & -0.81 \\
\hline & & 26488.47 & $9 / 2$ & 273.711 & 16.80 & 20.62 & -0.73 & -0.64 \\
\hline & & 26739.74 & $11 / 2$ & 275.607 & 4.55 & 5.58 & -1.29 & -1.20 \\
\hline & & 27410.47 & $7 / 2$ & 280.800 & 11.50 & 14.11 & -0.87 & -0.78 \\
\hline & & 27627.03 & $11 / 2$ & 282.518 & 1.95 & 2.39 & -1.63 & -1.54 \\
\hline & & 28989.22 & $7 / 2$ & 293.830 & 42.70 & 52.40 & -0.26 & -0.17 \\
\hline & & 29034.43 & $9 / 2$ & 294.221 & 48.10 & 59.03 & -0.21 & -0.12 \\
\hline & & 30019.63 & $7 / 2$ & 303.007 & 1.59 & 1.95 & -1.66 & -1.57 \\
\hline & & 30213.84 & $9 / 2$ & 304.801 & 14.90 & 18.29 & -0.68 & -0.59 \\
\hline & & 33045.60 & $9 / 2$ & 333.605 & 4.86 & 5.96 & -1.09 & -1.00 \\
\hline & & 33601.41 & $11 / 2$ & 339.910 & 3.34 & 4.10 & -1.24 & -1.15 \\
\hline & & 36741.56 & $7 / 2$ & 380.540 & 1.01 & 1.24 & -1.66 & -1.57 \\
\hline \multirow[t]{9}{*}{63041.81} & $5 / 2$ & 15199.38 & $5 / 2$ & 208.953 & 54.70 & 59.67 & -0.45 & -0.42 \\
\hline & & 15330.79 & $7 / 2$ & 209.529 & 58.60 & 63.93 & -0.41 & -0.38 \\
\hline & & 15691.45 & $5 / 2$ & 211.125 & 1.29 & 1.41 & -2.06 & -2.03 \\
\hline & & 15699.39 & $3 / 2$ & 211.160 & 2.66 & 2.90 & -1.75 & -1.72 \\
\hline & & 16947.08 & $7 / 2$ & 216.877 & 6.27 & 6.84 & -1.35 & -1.32 \\
\hline & & 17344.36 & $5 / 2$ & 218.762 & 3.75 & 4.09 & -1.57 & -1.54 \\
\hline & & 22444.51 & $5 / 2$ & 246.247 & 5.70 & 6.22 & -1.29 & -1.26 \\
\hline & & 22864.47 & $3 / 2$ & 248.821 & 1.98 & 2.16 & -1.74 & -1.71 \\
\hline & & 23853.58 & $7 / 2$ & 255.102 & 1.04 & 1.13 & -1.99 & -1.96 \\
\hline
\end{tabular}


Table 2. continued.

\begin{tabular}{|c|c|c|c|c|c|c|c|c|}
\hline \multirow{2}{*}{$\begin{array}{c}\text { Upper level }^{\mathrm{a}} \\
\left(\mathrm{cm}^{-1}\right)\end{array}$} & \multirow{2}{*}{$J$} & \multirow{2}{*}{$\begin{array}{c}\text { Lower level }^{\mathrm{a}} \\
\left(\mathrm{cm}^{-1}\right)\end{array}$} & \multirow{2}{*}{$J$} & \multirow{2}{*}{$\begin{array}{c}\lambda_{\text {air }} \\
(\mathrm{nm})\end{array}$} & \multicolumn{2}{|c|}{$g A\left(10^{7} \mathrm{~s}^{-1}\right)$} & \multicolumn{2}{|c|}{$\log (g f)$} \\
\hline & & & & & HFR & Corr. $^{\mathrm{b}}$ & HFR & Corr. $^{b}$ \\
\hline \multirow{31}{*}{63298.54} & \multirow{31}{*}{$7 / 2$} & 23934.63 & $5 / 2$ & 255.631 & 3.39 & 3.70 & -1.48 & -1.45 \\
\hline & & 24659.50 & $3 / 2$ & 260.459 & 1.61 & 1.76 & -1.79 & -1.76 \\
\hline & & 26041.36 & $7 / 2$ & 270.187 & 45.30 & 49.42 & -0.30 & -0.27 \\
\hline & & 26405.84 & $7 / 2$ & 272.875 & 12.50 & 13.64 & -0.86 & -0.83 \\
\hline & & 27627.86 & $3 / 2$ & 282.292 & 3.39 & 3.70 & -1.39 & -1.36 \\
\hline & & 27879.03 & $5 / 2$ & 284.308 & 1.28 & 1.40 & -1.81 & -1.78 \\
\hline & & 28877.24 & $5 / 2$ & 292.615 & 13.70 & 14.95 & -0.75 & -0.72 \\
\hline & & 28884.05 & $3 / 2$ & 292.674 & 19.40 & 21.16 & -0.60 & -0.57 \\
\hline & & 28989.22 & $7 / 2$ & 293.578 & 4.31 & 4.70 & -1.25 & -1.22 \\
\hline & & 29022.46 & $5 / 2$ & 293.865 & 3.24 & 3.53 & -1.38 & -1.35 \\
\hline & & 33146.56 & $7 / 2$ & 334.405 & 1.10 & 1.20 & -1.73 & -1.70 \\
\hline & & 37431.72 & $7 / 2$ & 390.361 & 1.16 & 1.27 & -1.58 & -1.55 \\
\hline & & 15199.38 & $5 / 2$ & 207.838 & 2.41 & 2.34 & -1.81 & -1.82 \\
\hline & & 15330.79 & $7 / 2$ & 208.407 & 7.00 & 6.81 & -1.34 & -1.35 \\
\hline & & 15427.90 & $9 / 2$ & 208.830 & 46.80 & 45.50 & -0.52 & -0.53 \\
\hline & & 16947.08 & $7 / 2$ & 215.675 & 4.18 & 4.06 & -1.54 & -1.55 \\
\hline & & 17344.36 & $5 / 2$ & 217.540 & 8.28 & 8.05 & -1.23 & -1.24 \\
\hline & & 23853.58 & $7 / 2$ & 253.442 & 3.41 & 3.32 & -1.48 & -1.49 \\
\hline & & 23934.63 & $5 / 2$ & 253.964 & 1.78 & 1.73 & -1.76 & -1.77 \\
\hline & & 24836.32 & $5 / 2$ & 259.918 & 7.87 & 7.65 & -1.10 & -1.11 \\
\hline & & 25112.57 & $5 / 2$ & 261.798 & 2.39 & 2.32 & -1.61 & -1.62 \\
\hline & & 25341.90 & $7 / 2$ & 263.380 & 1.28 & 1.24 & -1.88 & -1.89 \\
\hline & & 26041.36 & $7 / 2$ & 268.325 & 20.50 & 19.93 & -0.65 & -0.66 \\
\hline & & 26405.84 & $7 / 2$ & 270.976 & 1.90 & 1.85 & -1.68 & -1.69 \\
\hline & & 26488.47 & $9 / 2$ & 271.584 & 1.70 & 1.65 & -1.73 & -1.74 \\
\hline & & 27410.47 & $7 / 2$ & 278.562 & 8.35 & 8.12 & -1.01 & -1.02 \\
\hline & & 28877.24 & $5 / 2$ & 290.433 & 3.38 & 3.29 & -1.37 & -1.38 \\
\hline & & 28989.22 & $7 / 2$ & 291.381 & 32.90 & 31.99 & -0.38 & -0.39 \\
\hline & & 29699.47 & $5 / 2$ & 297.540 & 67.00 & 65.14 & -0.05 & -0.06 \\
\hline & & 30213.84 & $9 / 2$ & 302.167 & 1.53 & 1.49 & -1.68 & -1.69 \\
\hline & & 33045.60 & $9 / 2$ & 330.451 & 2.77 & 2.69 & -1.34 & -1.35 \\
\hline \multirow[t]{21}{*}{63392.75} & \multirow[t]{21}{*}{$5 / 2$} & 15199.38 & $5 / 2$ & 207.431 & 13.00 & 13.90 & -1.07 & -1.04 \\
\hline & & 15330.79 & $7 / 2$ & 207.999 & 21.60 & 23.09 & -0.85 & -0.82 \\
\hline & & 15699.39 & $3 / 2$ & 209.606 & 2.65 & 2.83 & -1.76 & -1.73 \\
\hline & & 17174.33 & $3 / 2$ & 216.296 & 5.21 & 5.57 & -1.44 & -1.41 \\
\hline & & 17344.36 & $5 / 2$ & 217.095 & 30.90 & 33.03 & -0.66 & -0.63 \\
\hline & & 22864.47 & $3 / 2$ & 246.667 & 18.20 & 19.46 & -0.78 & -0.75 \\
\hline & & 23853.58 & $7 / 2$ & 252.838 & 3.13 & 3.35 & -1.52 & -1.49 \\
\hline & & 23934.63 & $5 / 2$ & 253.357 & 8.75 & 9.35 & -1.07 & -1.04 \\
\hline & & 24137.91 & $3 / 2$ & 254.669 & 5.13 & 5.48 & -1.30 & -1.27 \\
\hline & & 24659.50 & $3 / 2$ & 258.099 & 3.98 & 4.25 & -1.40 & -1.37 \\
\hline & & 26041.36 & $7 / 2$ & 267.648 & 21.40 & 22.88 & -0.64 & -0.61 \\
\hline & & 27879.03 & $5 / 2$ & 281.498 & 2.17 & 2.32 & -1.59 & -1.56 \\
\hline & & 28877.24 & $5 / 2$ & 289.640 & 25.10 & 26.83 & -0.50 & -0.47 \\
\hline & & 28884.05 & $3 / 2$ & 289.697 & 5.38 & 5.75 & -1.17 & -1.14 \\
\hline & & 28989.22 & $7 / 2$ & 290.583 & 2.57 & 2.75 & -1.49 & -1.46 \\
\hline & & 29022.46 & $5 / 2$ & 290.864 & 2.53 & 2.70 & -1.50 & -1.47 \\
\hline & & 29699.47 & $5 / 2$ & 296.708 & 8.91 & 9.52 & -0.93 & -0.90 \\
\hline & & 33146.56 & $7 / 2$ & 330.525 & 1.35 & 1.44 & -1.65 & -1.62 \\
\hline & & 36741.56 & $7 / 2$ & 375.111 & 2.35 & 2.51 & -1.30 & -1.27 \\
\hline & & 37431.72 & $7 / 2$ & 385.084 & 1.46 & 1.56 & -1.49 & -1.46 \\
\hline & & 39243.69 & $3 / 2$ & 413.978 & 1.64 & 1.75 & -1.37 & -1.34 \\
\hline \multirow[t]{6}{*}{63497.73} & \multirow[t]{6}{*}{$9 / 2$} & 15330.79 & $7 / 2$ & 207.545 & 3.02 & 2.78 & -1.71 & -1.74 \\
\hline & & 15427.90 & $9 / 2$ & 207.964 & 3.39 & 3.12 & -1.66 & -1.69 \\
\hline & & 15447.04 & $11 / 2$ & 208.047 & 55.10 & 50.75 & -0.45 & -0.48 \\
\hline & & 16947.08 & $7 / 2$ & 214.752 & 7.62 & 7.02 & -1.28 & -1.31 \\
\hline & & 22980.45 & $11 / 2$ & 246.734 & 11.00 & 10.13 & -1.00 & -1.03 \\
\hline & & 23833.15 & $9 / 2$ & 252.038 & 2.19 & 2.02 & -1.68 & -1.71 \\
\hline
\end{tabular}


Table 2. continued.

\begin{tabular}{|c|c|c|c|c|c|c|c|c|}
\hline \multirow{2}{*}{$\begin{array}{c}\text { Upper level }^{\mathrm{a}} \\
\left(\mathrm{cm}^{-1}\right)\end{array}$} & \multirow{2}{*}{$J$} & \multirow{2}{*}{$\begin{array}{c}\text { Lower level }^{\mathrm{a}} \\
\left(\mathrm{cm}^{-1}\right)\end{array}$} & \multirow{2}{*}{$J$} & \multirow{2}{*}{$\begin{array}{c}\lambda_{\text {air }} \\
(\mathrm{nm})\end{array}$} & \multicolumn{2}{|c|}{$g A\left(10^{7} \mathrm{~s}^{-1}\right)$} & \multicolumn{2}{|c|}{$\log (g f)$} \\
\hline & & & & & HFR & Corr. $^{b}$ & HFR & Corr. ${ }^{b}$ \\
\hline & & 23853.58 & $7 / 2$ & 252.168 & 4.92 & 4.53 & -1.33 & -1.36 \\
\hline & & 24509.50 & $7 / 2$ & 256.411 & 1.15 & 1.06 & -1.95 & -1.98 \\
\hline & & 25341.90 & $7 / 2$ & 262.005 & 9.06 & 8.34 & -1.03 & -1.06 \\
\hline & & 26068.81 & $9 / 2$ & 267.094 & 6.08 & 5.60 & -1.19 & -1.22 \\
\hline & & 26405.84 & $7 / 2$ & 269.521 & 28.00 & 25.79 & -0.52 & -0.55 \\
\hline & & 26488.47 & $9 / 2$ & 270.123 & 7.95 & 7.32 & -1.06 & -1.09 \\
\hline & & 26739.74 & $11 / 2$ & 271.969 & 2.10 & 1.93 & -1.63 & -1.66 \\
\hline & & 27724.78 & $9 / 2$ & 279.458 & 3.71 & 3.42 & -1.36 & -1.39 \\
\hline & & 29034.43 & $9 / 2$ & 290.079 & 40.80 & 37.58 & -0.29 & -0.32 \\
\hline & & 30019.63 & $7 / 2$ & 298.616 & 58.00 & 53.42 & -0.11 & -0.14 \\
\hline & & 30391.61 & $11 / 2$ & 301.971 & 1.27 & 1.17 & -1.76 & -1.79 \\
\hline & & 33045.60 & $9 / 2$ & 328.290 & 23.90 & 22.01 & -0.41 & -0.44 \\
\hline & & 35406.26 & $11 / 2$ & 355.878 & 1.01 & 0.93 & -1.72 & -1.75 \\
\hline & & 36741.56 & $7 / 2$ & 373.639 & 3.06 & 2.82 & -1.20 & -1.23 \\
\hline & & 37431.72 & $7 / 2$ & 383.533 & 5.21 & 4.80 & -0.94 & -0.97 \\
\hline
\end{tabular}

${ }^{\mathrm{a}}$ From Nilsson and Pickering [13]. ${ }^{\mathrm{b}}$ Deduced from the measured lifetimes and the calculated HFR branching fractions.

\section{References}

1. C.M. Sikström, H. Nilsson, U. Litzén, A. Blom, H. Lindberg, J. Quant. Spectrosc. Radiat. Transf. 74, 355 (2002)

2. D.C. Morton, Astrophys. J. Suppl. 130, 403 (2000)

3. M. Jaschek, E. Brandi, Astron. Astrophys. 20, 233 (1972)

4. C.R. Cowley, M. Hartoog, A.P. Cowley, Astrophys. J. 194, 343 (1974)

5. S.J. Adelman, Astrophys. J. Suppl. 26, 1 (1973)

6. J.C. Brandt et al., Astron. J. 117, 1505 (1999)

7. P. Hannaford, R.M. Lowe, J. Phys. B 16, 4539 (1983)

8. C.M. Sikström, H. Pihlemark, H. Nilsson, U. Litzén, S. Johansson, Z.S. Li, H. Lundberg, J. Phys. B 34, 477 (2001)
9. P. Quinet, J. Phys. B 35, 19 (2002)

10. H. Lundberg, L. Engström, H. Hartman, H. Nilsson, P. Palmeri, P. Quinet, É. Biémont, J. Phys. B 43, 085004 (2010)

11. W. Zhang, Y.Y. Feng, J.X. Xu, P. Palmeri, P. Quinet, É. Biémont, Z.W. Dai, J. Phys. B 43, 205005 (2010)

12. S. Schiemann, W. Hogervorst, W. Ubachs, IEEE J. Quantum Electron. 34, 407 (1998)

13. H. Nilsson, J.C. Pickering, Phys. Scr. 67, 223 (2003)

14. R.D. Cowan, The Theory of Atomic Structure and Spectra (University of California Press, Berkeley, 1981)

15. P. Quinet, P. Palmeri, É. Biémont, M.M. McCurdy, G. Rieger, E.H. Pinnington, M.E. Wickliffe, J.E. Lawler, Mon. Not. R. Astron. Soc. 307, 934 (1999) 